[Supporting Information]

\title{
A self-powered gas sensor based on a photovoltaic cell and a colorimetric film with hierarchical micro/nano-structures
}

Kyungnam Kang ${ }^{a, \dagger}$, Jaeho Park ${ }^{a, \dagger}$, Byeongsu $\mathrm{Kim}^{b}, \mathrm{Kwangmin} \mathrm{Na}^{c}$, Incheol Cho ${ }^{a}$, Junsuk Rho ${ }^{d, e}$,

Daejong Yang ${ }^{*}$, , Jung-Yong Lee ${ }^{*}, b$ and Inkyu Park ${ }^{*}, a$

a Department of Mechanical Engineering, Korea Advanced Institute of Science and Technology

(KAIST), Daejeon 34141, Republic of Korea

${ }^{\mathrm{b}}$ School of Electrical Engineering, Korea Advanced Institute of Science and Technology

(KAIST), Daejeon 34141, Republic of Korea

${ }^{c}$ Graduate School of EEWS, Korea Advanced Institute of Science and Technology (KAIST),

Daejeon 34141, Republic of Korea

${ }^{\mathrm{d}}$ Department of Mechanical Engineering, Pohang University of Science and Technology

(POSTECH), Pohang 37673, Republic of Korea 
e Department of Chemical Engineering, Pohang University of Science and Technology (POSTECH), Pohang 37673, Republic of Korea

${ }_{\mathrm{f}}^{\mathrm{f}}$ Department of Mechanical and Automotive Engineering, Kongju National University, Cheonan, 31080, Republic of Korea

†These authors equally contributed to this work 


\section{Chemical reaction of TMPD with $\mathrm{NO}_{2}$ gas}

TMPD



TMPD $^{+}$



Two co-existing resonance modes

Figure S1. Schematic illustration of reduction-oxidation of a TMPD molecule by $\mathrm{NO}_{2}$ gas: When a TMPD molecule is exposed to $\mathrm{NO}_{2}$ gas, it is oxidized and turn into a TMPD ${ }^{+}$ion that shows blue color. The oxidized TMPD ions possess two co-existing resonance modes. 


\section{Numerical simulation for absorption spectra for different photoactive layers and thicknesses}

To estimate the absorption spectra of different photovoltaic materials in Figure $2 \mathrm{~b}$, we implemented the transfer matrix formalism simulation using a customized MATLAB code. All refractive indices of photovoltaic materials was experimentally obtained using the ellipsometry. Overall device structure for the optical simulation was composed of ITO $(75 \mathrm{~nm}) / \mathrm{ZnO}(30 \mathrm{~nm}) /$ photovoltaic layer $(50-250 \mathrm{~nm}) / \mathrm{MoO}_{3}(10 \mathrm{~nm}) / \mathrm{Ag}(150 \mathrm{~nm})$. We assumed that each layer is flat and there are no scattering effects. Furthermore, to identify the optimal thickness for the maximized photo-responsivities for each photoactive material, numerical simulation for shortcircuit current densities was performed. For the calculation, pre-obtained absorption spectra in Figure $2 \mathrm{~b}$ was utilized, and the short-circuit current densities for thicknesses from $50 \mathrm{~nm}$ to 250 $\mathrm{nm}$ with $1 \mathrm{~nm}$ increment were calculated as shown in Figure 2c. It should be noted a perfect charge extraction condition was assumed, which means that the internal quantum efficiency is $100 \%$. 


\section{Transmittance of PDMS with different structures without TMPD coating}
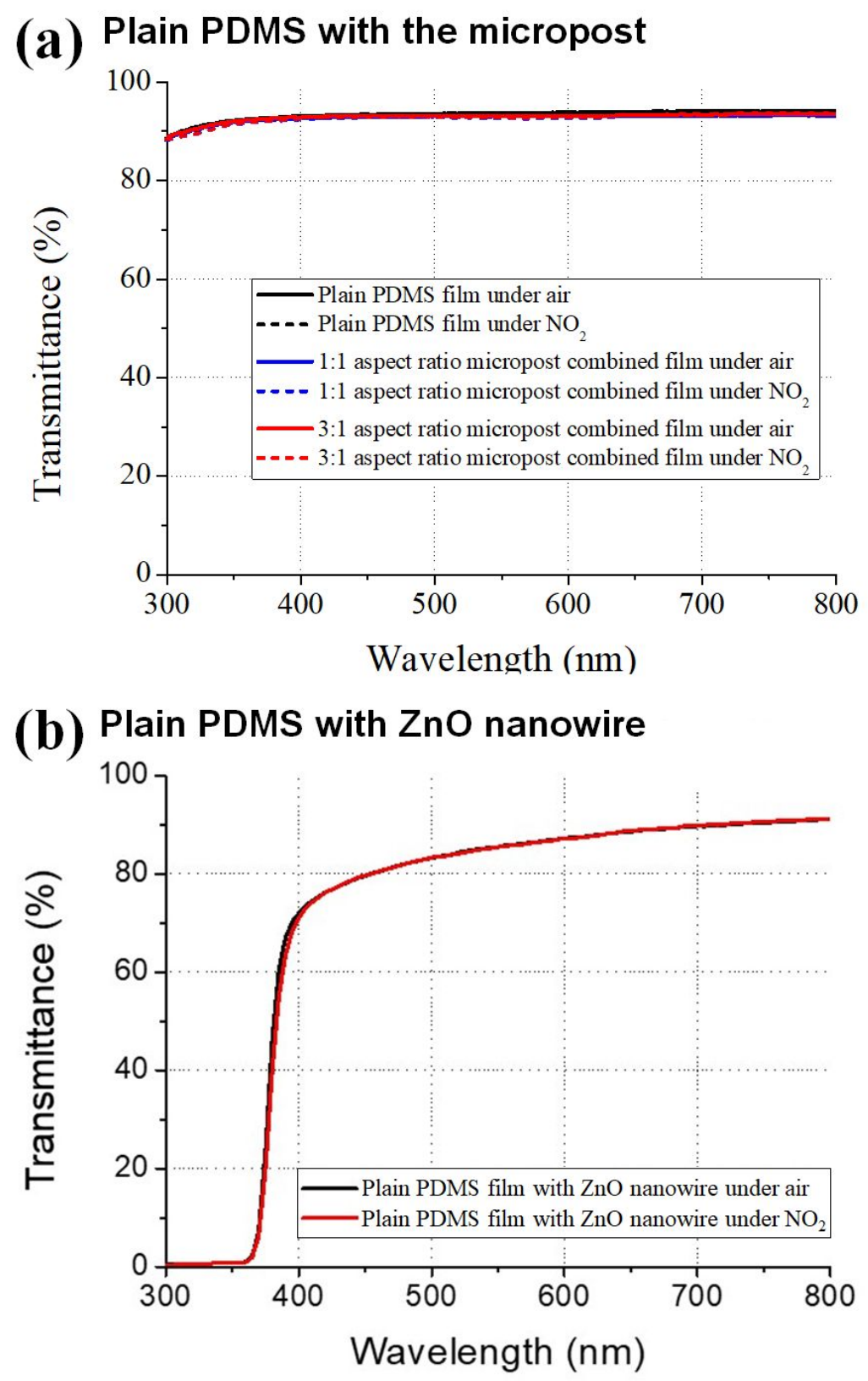

Figure S2. Transmittance of plain PDMS substrate, and microstructured PDMS (1:1 aspect ratio micropost array and 3:1 aspect ratio micropost array) substrate and plain PDMS substrate with $\mathrm{ZnO}$ nanowires before and after exposure to $20 \mathrm{ppm} \mathrm{NO}_{2}$ exposure for 30 minutes. 


\section{Sensor responses to $\mathrm{NO}_{2}$ with diffused and collimated light sources}

(a) Collimated light source
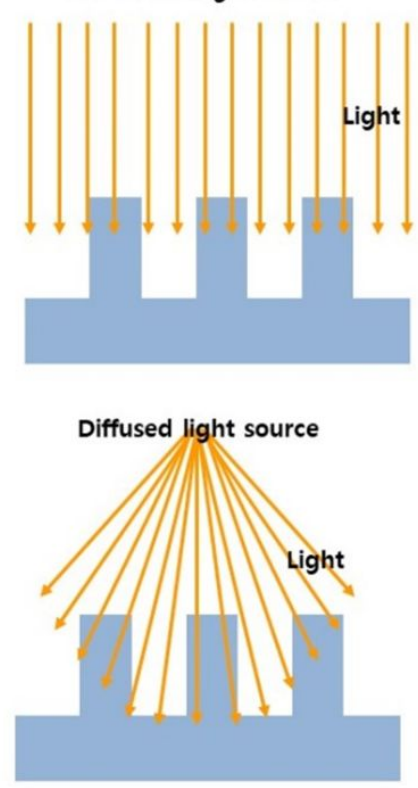

(b)



Figure S3. (a) Schematic of collimated and diffused light sources and (b) Sensor response of a self-powered gas sensor to $\mathrm{NO}_{2}$ gas using collimated and diffused light sources. Both illumination types resulted in similar sensor responses. 


\section{5. $\mathrm{NO}_{2}$ gas sensing performance under various light intensities}

(a)

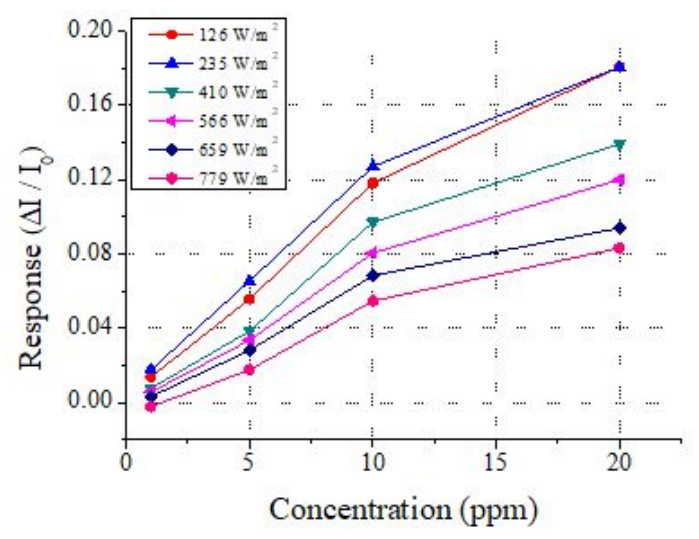

(c)

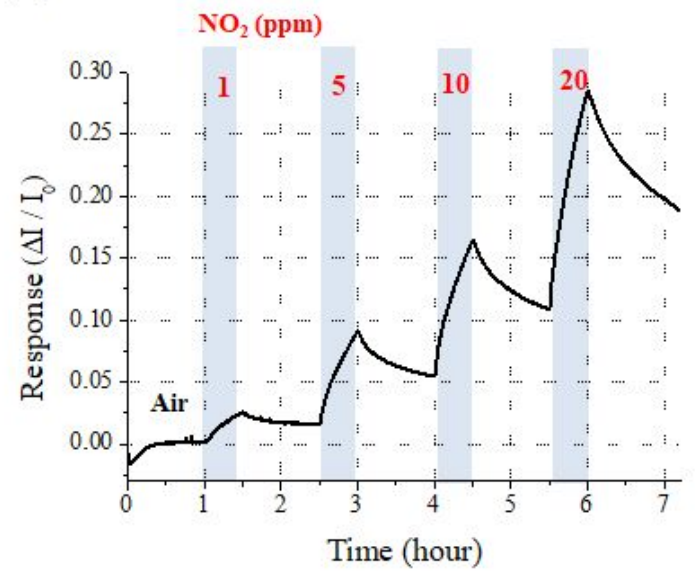

(b)

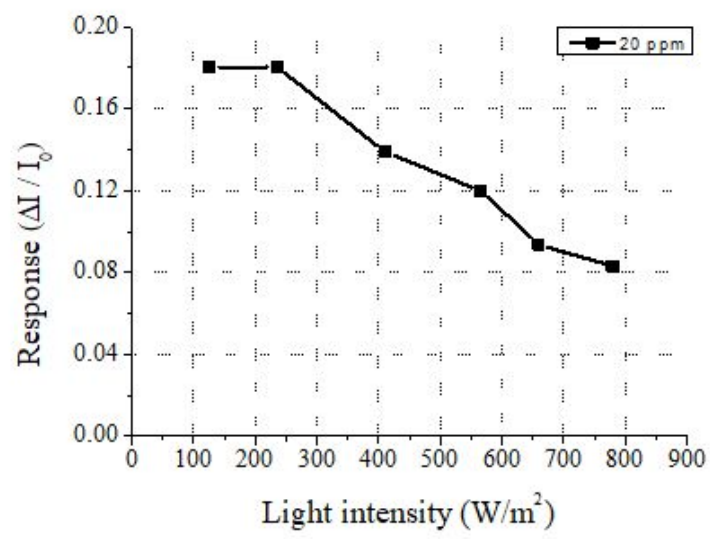

(d)



Figure S4. (a) $\mathrm{NO}_{2}$ gas sensing performance of a self-powered gas sensor under various intensities of light generated by the solar illuminator. (b) Response to $20 \mathrm{ppm} \mathrm{NO}_{2}$ under various light intensities. (c-d) Response vs. time (c) and response vs. concentration (d) of self-powered gas sensor with a dim indoor LED lamp (light intensity=19 W/m²). 